\title{
Intranasal administration of a-synuclein preformed fibrils triggers microglial iron deposition in the substantia nigra of Macaca fascicularis
}

Jian-Jun Guo ${ }^{1}$, Feng Yue ${ }^{2}$, Dong-Yan Song ${ }^{1}$, Luc Bousset ${ }^{3}$, Xin Liang ${ }^{4}$, Jing Tang ${ }^{4}$, Lin Yuan ${ }^{5}$, Wen Li, ${ }^{5,6}$, Ronald Melki ${ }^{3}$, Yong Tang ${ }^{4}$, Piu Chan ${ }^{2}$, Chuang Guo ${ }^{1}$ and Jia-Yi Li (i) ${ }^{1,5,6}$

\begin{abstract}
Iron deposition is present in main lesion areas in the brains of patients with Parkinson's disease (PD) and an abnormal iron content may be associated with dopaminergic neuronal cytotoxicity and degeneration in the substantia nigra of the midbrain. However, the cause of iron deposition and its role in the pathological process of PD are unclear. In the present study, we investigated the effects of the nasal mucosal delivery of synthetic human a-synuclein (a-syn) preformed fibrils (PFFs) on the pathogenesis of PD in Macaca fascicularis. We detected that iron deposition was clearly increased in a time-dependent manner from 1 to 17 months in the substantia nigra and globus pallidus, highly contrasting to other brain regions after treatments with a-syn PFFs. At the cellular level, the iron deposits were specifically localized in microglia but not in dopaminergic neurons, nor in other types of glial cells in the substantia nigra, whereas the expression of transferrin (TF), TF receptor 1 (TFR1), TF receptor 2 (TFR2), and ferroportin (FPn) was increased in dopaminergic neurons. Furthermore, no clear dopaminergic neuron loss was observed in the substantia nigra, but with decreased immunoreactivity of tyrosine hydroxylase $(\mathrm{TH})$ and appearance of axonal swelling in the putamen. The brain region-enriched and cell-type-dependent iron localizations indicate that the intranasal a-syn PFFs treatment-induced iron depositions in microglia in the substantia nigra may appear as an early cellular response that may initiate neuroinflammation in the dopaminergic system before cell death occurs. Our data suggest that the inhibition of iron deposition may be a potential approach for the early prevention and treatment of PD.
\end{abstract}

\section{Introduction}

Parkinson's disease (PD) is a common neurodegenerative disease. The motor symptoms of PD include resting tremor, bradykinesia, rigidity, and postural abnormalities $^{1}$. PD patients can also exhibit different nonmotor symptoms, such as constipation ${ }^{2}$, depression ${ }^{3}$, and cognitive decline $\mathrm{e}^{4}$, which usually precede the motor

\footnotetext{
Correspondence: Chuang Guo (guoc@mail.neu.edu.cn) or Jia-Yi Li (lijiayi@cmu. edu.cn)

${ }^{1}$ Institute of Neuroscience, College of Life and Health Sciences, Northeastern University, Shenyang 110819, China

2Beijing Key Laboratory of Parkinson's Disease, National Clinical Research Center for Geriatric Disorders, Department of Neurobiology, Xuanwu Hospital of Capital Medical University, Beijing 100053, China

Full list of author information is available at the end of the article

Edited by F. Blandini
}

symptoms. Clinically, there are no effective early diagnostic or therapeutic tools for PD. Thus, robust biomarkers for the identification of PD are essential for early diagnosis and interventions ${ }^{5}$.

The main pathological features of PD include the degeneration of dopaminergic neurons in the substantia nigra and the appearance of Lewy bodies, Luria-Bertani medium (LBs) in surviving neurons ${ }^{6}$. The protein $\alpha$ synuclein ( $\alpha$-syn) is a constitutive component of LBs that is normally a monomeric protein under physiological conditions but forms amyloid fibrils under pathological conditions. The aggregation of $\alpha$-syn is closely associated with PD progression. Emerging evidence has shown that the pathological propagation of $\alpha$-syn in the brain may contribute to PD progression ${ }^{7}$. Multiple risk factors have

\section{(c) The Author(s) 2021}

(c) (i) Open Access This article is licensed under a Creative Commons Attribution 4.0 International License, which permits use, sharing, adaptation, distribution and reproduction in any medium or format, as long as you give appropriate credit to the original author(s) and the source, provide a link to the Creative Commons license, and indicate if changes were made. The images or other third party material in this article are included in the article's Creative Commons license, unless indicated otherwise in a credit line to the material. If material is not included in the article's Creative Commons license and your intended use is not permitted by statutory regulation or exceeds the permitted use, you will need to obtain permission directly from the copyright holder. To view a copy of this license, visit http://creativecommons.org/licenses/by/4.0/. 
been shown to jointly contribute to the development of $\mathrm{PD}$, including genetic and environmental factors ${ }^{8-10}$.

Iron is among the most essential trace elements in the human body. Excessive amounts of iron have toxic effects on the nervous system. The factors associated with an increased total iron concentration mainly include aging, the inflammatory response, changes in iron balance, redistribution of iron in the brain, and increased blood-brain barrier permeability ${ }^{11,12}$. Many studies have demonstrated that the iron content in the substantia nigra of PD patients is significantly increased, accompanied by changes in iron metabolism. Therefore, it has been proposed that iron may cause neuronal death by promoting the production of hydroxyl radicals ${ }^{13,14}$. Both epidemiological investigations and related experimental studies have shown that iron is closely associated with the pathogenesis of $\mathrm{PD}^{15}$. The accumulation of iron in neurons may cause apoptotic damage ${ }^{16}$ and the accumulation of iron in glial cells induces an inflammatory state by increasing the release of proinflammatory cytokines, leading to neuroinflammation ${ }^{17}$. Semiquantitative histochemical assays of postmortem PD brains have shown that $\mathrm{Fe}^{3+}$ is significantly increased in the substantia nigra pars compacta. Indeed, increased iron deposits in neurons and glia and the proliferation of iron-containing microglia have been reported in $\mathrm{PD}^{18-20}$. Moreover, ferric iron has been shown to promote $\alpha$-syn aggregation in vitro ${ }^{21,22}$. In the in situ detection of redox-active iron, neurons in the substantia nigra pars compacta of patients with PD showed strong LBs labeling, which is consistent with the PD oxidative stress hypothesis ${ }^{23}$. In patient brains with more severe neuronal loss, the content of redox-active iron is even higher ${ }^{24}$. Furthermore, an increase in the iron content in the substantia nigra can be observed even in the early stages of $\mathrm{PD}^{25}$, suggesting that iron accumulation can be used as an indicator to detect the pathological processes of PD and provide a new target for PD prevention and treatment.

To date, how different brain regions maintain iron concentrations under normal physiological conditions and how the iron distribution changes with inflammatory damage remain unclear. Whether iron deposition occurring in neurodegenerative diseases is a primary event or a secondary effect remains to be elucidated ${ }^{26}$. Previous studies investigating iron regulation have heavily relied on healthy rodents, but iron regulation in rodents differs from that in humans. Therefore, to study the pathogenesis of PD, we generated a novel animal model in which $\alpha$-syn preformed fibrils (PFFs) were directly administered on the surface of the olfactory epithelium and additionally injected into the olfactory submucosa of monkeys. Robust iron deposits appeared in the substantia nigra and globus pallidus of the animals exposed to $\alpha$-syn PFFs. At the cellular level, the iron deposition appeared specifically localized in microglia, rather than in dopaminergic neurons nor other types of glia. Although we did not detect clear dopaminergic neuronal loss in the substantia nigra, we observed signs of neurodegenerative alterations in dopaminergic terminals in the putamen, suggesting that iron deposition in microglia may precede dopaminergic neuronal death.

\section{Materials and methods \\ Monkeys used in the project}

In total, 12 male cynomolgus monkeys (Macaca fascicularis) ranging in age from 6 to 10 years (similar to human adulthood) were selected and randomly divided into the following two groups: $\alpha$-syn PFFs groups $(n=9)$ and control group treated with phosphate-buffered saline (PBS) $(n=3)$. The monkeys were maintained at the Grandforest Primate Breeding Co. Ltd, Guangxi, China, and all experimental animals had detailed birth records and quarantine certificates.

In the monkeys exposed to the $\alpha$-syn PFFs, $20 \mu \mathrm{l}$ of $1 \mu \mathrm{g} /$ $\mu \mathrm{l} \alpha$-syn PFFs was smeared unilaterally (right side) on the olfactory epithelial mucosa, followed by injection $(20 \mu \mathrm{l})$ into the olfactory epithelium. The total volume of the two administrations was $40 \mu$ l. The treatment was performed once a week for 4 weeks. The animals were killed and brain samples were collected 1, 4, and 17 months after $\alpha$ syn administration; there were three monkeys in each time point (Supplementary Fig. 1).

Under deep anesthesia with pentobarbital sodium, the heart of each monkey was exposed and intracardially perfused with $2-31$ of $0.01 \mathrm{M}$ PBS, followed by $4 \%$ paraformaldehyde (PFA) solution. Some monkey brains were perfused with PBS alone, removed, and cut into 2 or $4 \mathrm{~mm}$-thick brain slices. The $2 \mathrm{~mm}$-thick brain slices were immediately placed on dry ice and stored in a $-80^{\circ} \mathrm{C}$ freezer until use and the $4 \mathrm{~mm}$-thick brain slices were fixed in $4 \%$ PFA for the paraffin-embedding processes. The remaining monkey brains were cut into anterior, middle, and posterior blocks and directly placed in $4 \%$ PFA for post-fixation over two nights. The fixed monkey brains were cut into $40 \mu \mathrm{m}$-thick sections.

\section{Generation of a-syn PFFs}

The expression and purification of human wild-type (WT) $\alpha$-syn was performed as previously described ${ }^{27}$. Briefly, the Escherichia coli strain BL21 (DE3) (Stratagene, La Jolla, CA, USA) was transformed with expression vector pET3a encoding WT $\alpha$-syn and the bacteria were grown in LB medium to an optical density of 0.8 . $\alpha$-Syn expression was induced by $0.5 \mathrm{mM}$ isopropyl $\beta$-D-1-thiogalactopyranoside for $3 \mathrm{~h}$. The cells were lysed by sonication and the cell lysates were clarified by centrifugation at $14,000 \times g$ for $30 \mathrm{~min}$. $\alpha$-Syn was precipitated by $50 \%$ ammonium sulfate at $4{ }^{\circ} \mathrm{C}$. The solution was centrifuged at $4000 \times g$ for $30 \mathrm{~min}$ at $4{ }^{\circ} \mathrm{C}$ and the resulting pellet was 
resuspended in $10 \mathrm{mM}$ Tris $\mathrm{pH}$ 7.5. The solution was loaded onto a diethylaminoethyl cellulose column, which was eluted by a gradient of $0-500 \mathrm{mM} \mathrm{NaCl}$, and the fractions containing $\alpha$-syn (eluted at $200 \mathrm{mM} \mathrm{NaCl}$ ) were heated to $75^{\circ} \mathrm{C}$ for $20 \mathrm{~min}$. Then, the solution was clarified by centrifugation at $14,000 \times g$, loaded onto a Superdex 75 HiLoad 26/60 column (GE Healthcare), equilibrated, and eluted in $50 \mathrm{mM}$ Tris- $\mathrm{HCl}(\mathrm{pH} 7.5)$ and $150 \mathrm{mM} \mathrm{KCl}$. Pure $\alpha$-syn $(0.2-0.5 \mathrm{mM})$ in $50 \mathrm{mM}$ Tris$\mathrm{HCl}(\mathrm{pH} 7.5)$ and $150 \mathrm{mM} \mathrm{KCl}$ was filtered through sterile $0.22 \mu \mathrm{m}$ filters and stored at $-80^{\circ} \mathrm{C}$. The $\alpha$-syn concentration was determined spectrophotometrically using an extinction coefficient of $5960 \mathrm{M}^{-1} \mathrm{~cm}^{-1}$ at $280 \mathrm{~nm}$. For the fibril formation, $\alpha$-syn was incubated at $37^{\circ} \mathrm{C}$ under continuous shaking in an Eppendorf Thermomixer set at 600 r.p.m. The assembly was continuously monitored in a Cary Eclipse spectrofluorometer (Varian, Inc., Palo Alto, CA, USA) in the presence of Thioflavin $\mathrm{T}$ at an excitation wavelength of 440, an emission wavelength of 480, and an average time of $1 \mathrm{~s}$.

\section{Immunohistochemistry}

Brains post-fixed in 4\% PFA were transferred to a $15 \%$ sucrose solution and then a $30 \%$ sucrose solution for gradient infiltration. The brains were cut into $40 \mu \mathrm{m}$-thick sections using a microtome (SM2010R, Leica, Germany). The slices were rinsed, immersed in a mixture of $3 \% \mathrm{H}_{2} \mathrm{O}_{2}$ and methyl alcohol for 20 min to block endogenous peroxidase activity, and then blocked in $10 \%$ normal serum obtained from an animal of the same species that produced the secondary antibodies. The sections were incubated with rabbit anti-Iba1 (Wako, 1:800), mouse antityrosine hydroxylase (TH, ImmunoStar, 1:2000), and Ser129 phosphorylated $\alpha$-syn antibody (Abcam, 1:2000) overnight at $4{ }^{\circ} \mathrm{C}$, followed by rinsing three times with PBS. Then, the sections were incubated with a biotinylated secondary antibody against the primary antibodies for $2 \mathrm{~h}$, followed by incubation with an $\mathrm{ABC}$ kit for $1 \mathrm{~h}$. The sections were colorized with 3, 3'-diaminobenzidine (DAB) or alkaline phosphatase staining was performed using the Red Alkaline Phosphatase Substrate kit (SK5100 , Vector Labs, USA) according to the manufacturer's instructions. Finally, the sections were mounted on glass slides. After dehydration and clearance with xylene, the sections were mounted with coverslips and observed under a microscope (DM4000B, Leica, Germany).

For paraffin-embedded tissues, paraffin sections $(5 \mu \mathrm{m}$ thick) were deparaffinized with xylene and then sequential decreasing concentrations of ethanol before going through the immunohistochemistry procedure described above.

\section{Perl's-DAB ferric iron staining}

The frozen sections were rinsed with distilled water for $10 \mathrm{~min}$ and a mixture of $2 \% \mathrm{~K}_{4}\left[\mathrm{Fe}(\mathrm{CN})_{6}\right](10016818$,
Sinopharm Chemical Reagent Co., Ltd, China) and 2\% $\mathrm{HCl}$ was added dropwise to the sections. The sections were then incubated at room temperature for $30 \mathrm{~min}$ and washed three times with PBS. Then, eosin staining solution (C0109, Beyotime, China) was used to counterstain the cytoplasm, or the sections were counterstained with $\mathrm{DAB}$ for $3 \mathrm{~min}$. After dehydration through ethanol and clearing in xylene, the sections were mounted on glass slides. The sections were examined under a Leica brightfield microscope (DM4000B, Leica, Germany). Blue or brown granules were regarded as positive staining of iron ions in the tissue section.

\section{Immunofluorescence}

The brain sections were incubated with normal serum from the animal species that produced the secondary antibodies for $30 \mathrm{~min}$ and then incubated with mouse anti-transferrin (TF; Santa Cruz, 1:1000), mouse anti-TF receptor 1 (TFR1; Thermo, 1:500), rabbit anti-TF receptor 2 (TFR2; Abcam, 1:200), rabbit anti-ferroportin (FPn; Thermo, 1:1000), rabbit anti-ferritin (Abcam, 1:500), goat anti-Iba1 (Abcam, 1:200), and mouse anti-TH (ImmunoStar, 1:2000) overnight at $4{ }^{\circ} \mathrm{C}$. Subsequently, the sections were incubated with DyLight 594-labeled goat antimouse IgG or goat anti-rabbit IgG. Finally, the sections were labeled with 4' 6-diamidino-2-phenylindole (DAPI) (H-1500, Vector Labs, USA), mounted with antifluorescence quenching mounting medium, and observed under a confocal laser scanning microscope (SP8, Leica, Germany).

\section{Atomic absorption spectrometry}

The brain tissue samples were accurately weighed with an analytical balance. The samples were treated with $200 \mu \mathrm{l}$ of nitric acid (Sigma, purity $\geq 90 \%$ ) at $100{ }^{\circ} \mathrm{C}$ for $30 \mathrm{~min}$. Once the samples cooled to room temperature, they were diluted to $5 \mathrm{ml}$ with $1 \%$ nitric acid. A flame atomic absorption spectrometer (ZEEnit700P, Analytik Jena, Germany) was used to detect the iron level.

\section{Stereological analyses of dopaminergic neurons in the substantia nigra}

The stereology equipment consisted of a microscope (Olympus) with a controller that controls the field of view of the mirror to move precisely on the $X$ and $Y$ axes, a camera connected to a computer monitor, a pointer that accurately measures the depth of focus, a microcomputer (ProScan, UK), and a $\times 100$ objective lens capable of performing accurate counting. Under the $\times 100$ oil mirror, the stereological unbiased counting frame, the test frame, is superimposed on the tissue image. Stereological methods were used to investigate the number of neurons in the substantia nigra and, ventral tegmental area (VTA). 
The total number of neurons in the substantia nigra region was calculated according to the following formula:

$$
N(\text { Total })=\sum \mathrm{Q}-\times 1 / \mathrm{ssf} \times 1 / \operatorname{asf} \times 1 / \text { tsf }
$$

" $\Sigma Q^{-"}$ represents the total number of neurons in the substantia nigra; "ssf" represents the sampling score of the tissue section, i.e., 1/19; "asf" represents the area sampling fraction of the substantia nigra region within the tissue section, i.e., 2\%; and "tsf" represents the thickness sampling fraction $(\mathrm{H} / \mathrm{T})$ of the substantia nigra region within the tissue section, where " $\mathrm{T}$ " is the average thickness of the tissue section $(\mu \mathrm{m})$ and " $\mathrm{H}$ " is the test height of the optical stereo frame.

\section{Quantification and statistical analyses}

To quantify the iron deposition in different brain regions and compare among different groups, we measured the optical density of the iron deposition in images taken under $\times 40$ objective with Image-Pro Plus software. To quantify the appearance of iron-containing microglia in the doublelabeled brain sections (after staining with iron dye $\mathrm{K}_{4}[\mathrm{Fe}$ $\left.(\mathrm{CN})_{6}\right]$ and Iba1-immunohistochemistry), we randomly selected ten regions per section of the respective brain regions studied and counted the iron-staining Iba1-positive microglia against the total Iba1-positive microglia in the same area, and the proportion of the level of iron deposited in microglia was calculated. To quantify the change in the number of dopaminergic neurons containing iron-related proteins, we counted the number of dopaminergic neurons in the images taken under a $\times 20$ objective where $\mathrm{TH}$ colocated with TF, TFR1, TFR2, or FPn, we randomly selected ten regions per section in the substantia nigra. For the quantification of TH-positive nerve terminals in the dorsolateral part of the putamen, we measured the intensities of the $\mathrm{TH}$ immunoreactivity in the images taken under $\times 40$ objective and randomly selected ten regions per section. The results were presented as the mean values $\pm \mathrm{SEM}$. One-way analysis of variance (ANOVA) was used to evaluate the treatment groups compared with the control group. Tukey's multiple comparisons test after two-way ANOVA was used to evaluate the difference between the left and right hemispheres using GraphPad Prism 8.0 software. A $p$-value $<0.05$ was used throughout this study to indicate the significance.

\section{Results}

Iron deposition was increased in the nigra-striatal system after the a-syn PFFs treatment

Previous studies have shown that iron deposition is highly associated with dopaminergic neuron cytotoxicity and degeneration in PD patients and animal models ${ }^{28-30}$. To explore whether exogenously delivered $\alpha$-syn PFFs treatment (via the olfactory epithelium) can alter iron deposition in the dopaminergic system, we first performed
Perl's chemical staining for ferric iron ion localization in monkey brain sections. As shown in Fig. $1 \mathrm{~A}-\mathrm{H}$, we observed a robust increase in iron deposition in the substantia nigra in response to the $\alpha$-syn PFFs treatment. Compared to the control (PBS treated) monkeys, the monkeys at 1 month after the treatment with $\alpha$-syn PFFs exhibited significant increase of iron deposition in the ipsilateral (right) side of the substantia nigra, and the iron deposition severity increased significantly in a timedependent manner from 1 to 17 months (Fig. 1I). Notably, we also detected the increased iron deposition in the contralateral side of the substantia nigra to $\alpha$-syn PFFs delivery (Fig. 1J), whereas there were statistically significant differences between the two sides of the brain at 17 months after $\alpha$-syn PFFs treatment (Fig. 1K).

As shown in Fig. 2, consistent changes similar to those in the substantia nigra were observed in the globus pallidus (Fig. 2A-J), but there was no statistically significant difference between the ipsilateral and contralateral sides of the brain (Fig. 2K). Moreover, we detected that the increasing tendency of iron deposition in the caudate nucleus (Supplementary Fig. 2a) was similar to that in the globus pallidus, but the degree was very mild. Interestingly, there was no obvious iron deposition in the hippocampus (Supplementary Fig. 2b), entorhinal cortex (Supplementary Fig. 2c), and olfactory bulb (Supplementary Fig. 2d), compared with the control monkeys after $\alpha$ syn PFFs treatment.

It was well-known that Perl's chemical staining visualized the ferric iron, as shown in Figs. 1 and 2, to examine the presence and potential changes of ferrous iron in response to $\alpha$-syn PFFs treatment, we performed Turnbull staining. It appeared that the staining intensity of ferrous iron in the substantia nigra and globus pallidus was very weak, and mainly in the tissues around blood vessels, and there was no clear increase in response to $\alpha$-syn PFF treatment (Supplementary Fig. 3a, b).

\section{Iron contents in different brain regions after the a-syn PFFs treatment}

To further validate the changes of iron contents in different brain regions following different treatments, we selected the substantia nigra, globus pallidus, prefrontal cortex, and hippocampus, and used atomic absorption spectrometry (AAS) to measure the iron content. Compared to the control monkeys, it was evident that the iron content increased by one- to twofolds in the substantia nigra and globus pallidus in the monkeys treated with the $\alpha$-syn PFFs, and the iron content increased in a timedependent manner from 1 to 17 months (Fig. 3A, B). There were no statistical differences in the iron content of hippocampus between $\alpha$-syn PFFs treatment and control (Fig. 3D), whereas the iron content in the prefrontal cortex of monkeys treated with $\alpha$-syn PFFs for 17 months 


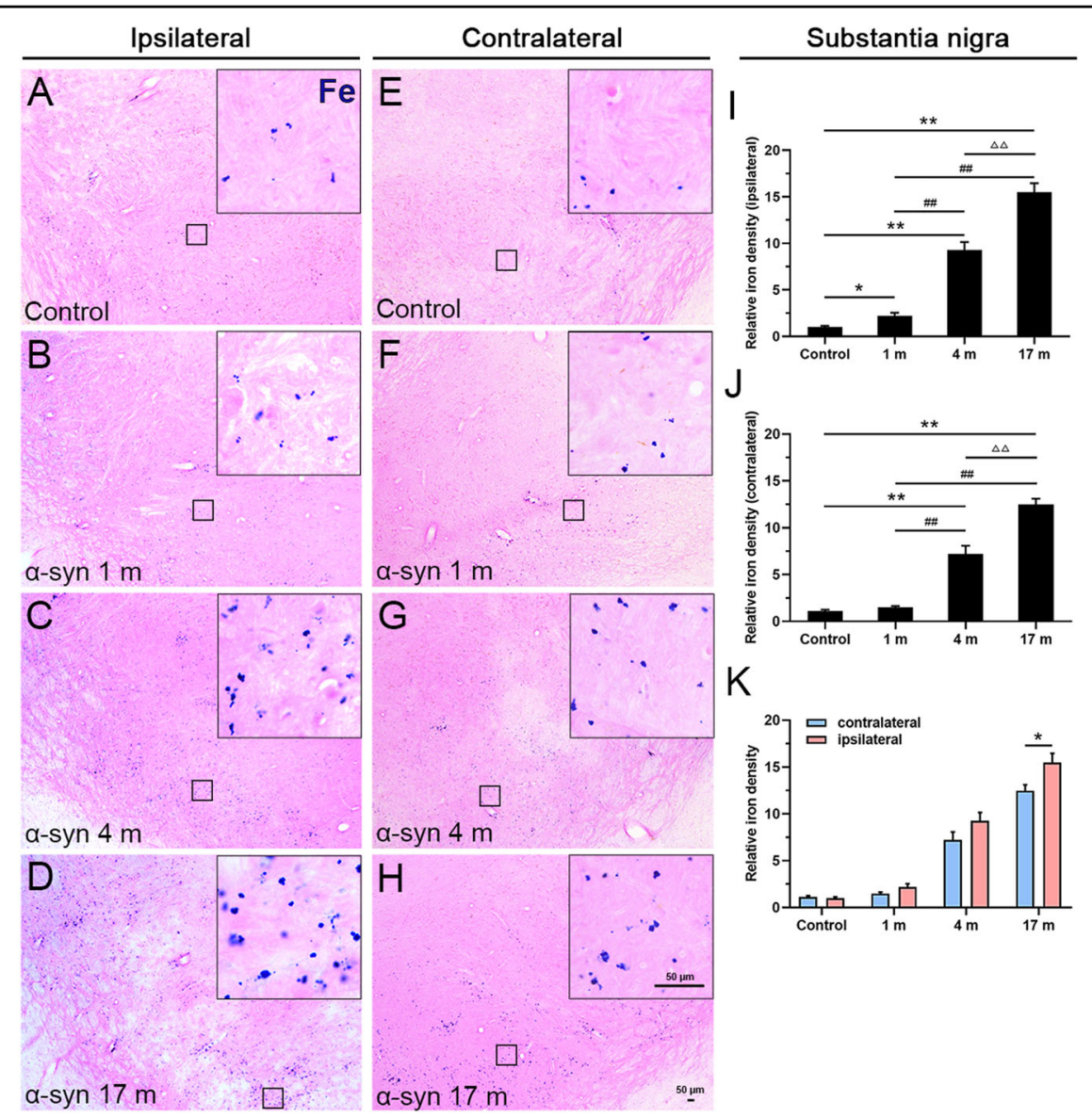

Fig. 1 Ferric iron deposition in the substantia nigra. Chemical staining of iron ions showed a gradual increase in iron deposition, 1 (B), 4 (C), and 17 (D) months after the treatment with a-syn PFFs, compared to the control $(\mathbf{A})$ in the ipsilateral side. $\mathbf{E}-\mathbf{H}$ Chemical staining of iron ions in the control $(\mathbf{E})$ and $1(\mathbf{F}), 4(\mathbf{G})$, and 17 months $(\mathbf{H})$ after treatment with a-syn PFFs in the contralateral side. The inset images depict the enlarged small boxes in $\mathbf{A}-\mathbf{H}$. I-K Quantitative analyses of the iron deposition density in the substantia nigra (ipsilateral side: I; contralateral side: J; both sides: K). Iron deposition staining levels were quantified with Image-Pro Plus software as the relative optical density above the background. $\mathbf{a}$-Syn, a-synuclein. The data are presented as the mean \pm SEM of three independent experiments. $n=3$ in each group and time points. ${ }^{*} p<0.05$ and ${ }^{* *} p<0.01$ vs. control group, ${ }^{\# \#} p<0.01$ vs. 1 -month group, ${ }^{\Delta} p<0.01$ vs. 4 -month group, one-way ANOVA (I, J). ${ }^{*} p<0.05$ vs. contralateral group, Tukey's multiple comparisons test after two-way ANOVA (K). Scale bars: $50 \mu \mathrm{m}$ in $\mathbf{A}-\mathbf{H}$ and $50 \mu \mathrm{m}$ in the insets.

was significantly increased compared with the control (Fig. 3C). The AAS data are consistent with the observations of the chemical iron staining. In addition, we observed that the expression of ferritin in the substantia nigra of $\alpha$-syn PFF-treated monkeys was more than that of the control monkey in a time-dependent manner. Interestingly, ferritin was mainly localized in microglia (Fig. $3 \mathrm{E}$ ), the expression of ferritin in the dopaminergic neurons was low, even though it was colocalized with $\mathrm{TH}$ in the 17-month monkeys after $\alpha$-syn treatment (Fig. 3F).

\section{Selectively increased iron deposition in microglia}

It has been shown that excess iron is toxic to dopaminergic neurons ${ }^{31,32}$. Next, we examined the cell-typespecific localization of iron deposition, e.g., where the iron deposition was the most enriched. As shown by the chemical staining of iron ions in the substantia nigra (Fig. 1A-H), the level of iron was increased in response to $\alpha$-syn PFFs treatment. Surprisingly, the double-labeling by immunostaining for $\mathrm{TH}$, which is the key enzyme involved in dopamine synthesis, and the chemical staining of iron ions showed limited or no overlap (colocalization) between $\mathrm{TH}$ and iron deposits in dopaminergic neurons, iron deposition mainly exists in the dorsolateral area of the substantia nigra (Fig. 4A). Iron deposits were not localized in astrocytes (GFAP-positive) (Fig. 4B), nor in oligodendrocytes (Olig2-positive) (Fig. 4C). In contrast, we observed that many microglia in the substantia nigra (Fig. 5A-H) and globus pallidus (Fig. 6A-H) exhibited robust iron deposition. On the ipsilateral side, iron deposition significantly increased 1 month after $\alpha$-syn PFFs treatment compared to the control group, and the 


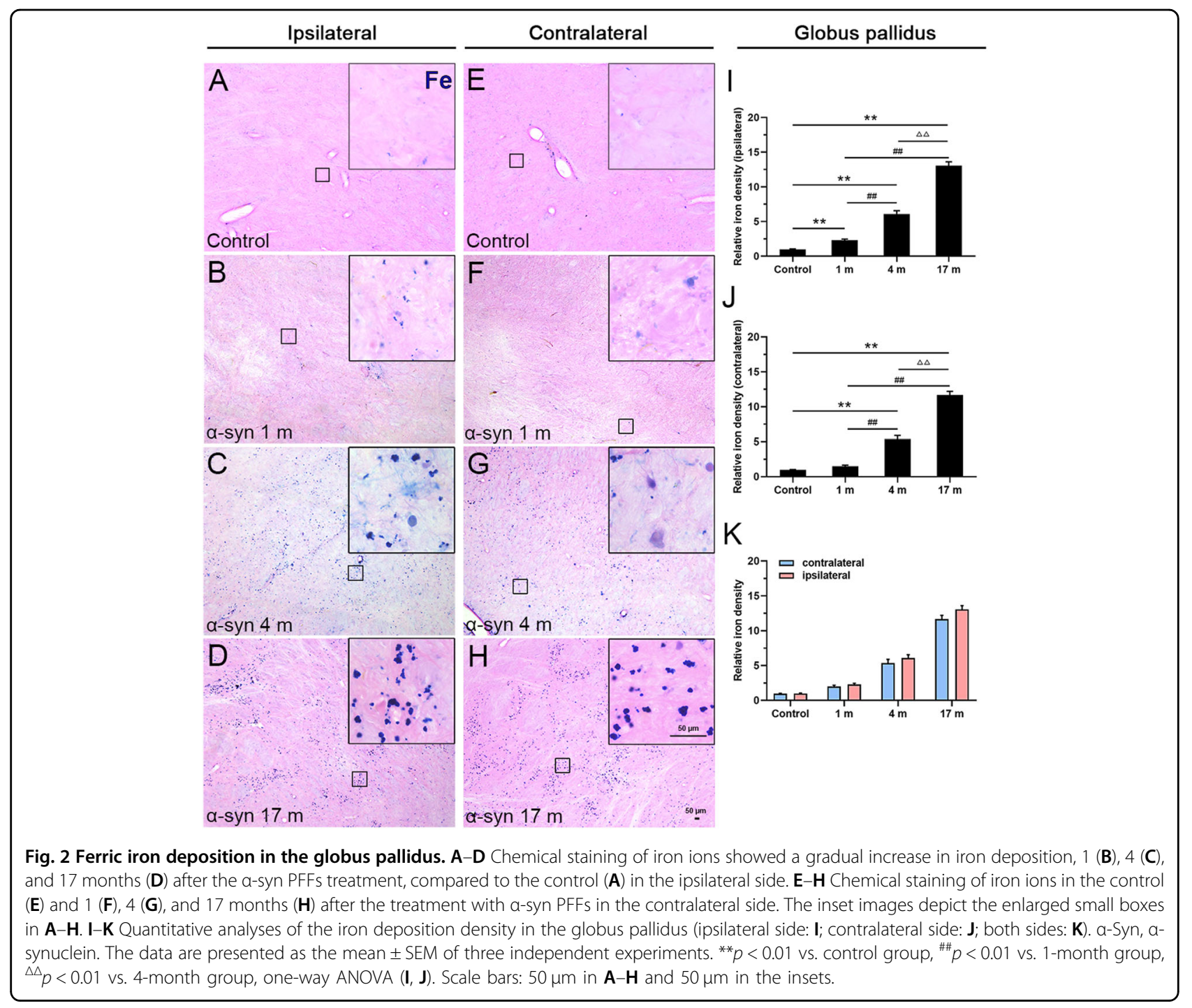

iron deposition in microglia severity increased further in a time-dependent manner from 1 to 17 months (Figs. 5I and 6I). Moreover, we detected that the extent of the increase in iron deposition in microglia in the substantia nigra and globus pallidus in the contralateral side to the $\alpha$-syn PFFs delivery (Figs. 5J and 6J) was similar to that in the ipsilateral side (Figs. 5I and 6I). In the 1-month treatment group, the increase of iron deposition in the contralateral side was not statistically significant in microglia in the substantia nigra and globus pallidus. Moreover, the iron deposition increased to a large extent in the ipsilateral side than that in the contralateral side of the substantia nigra at 17 months after $\alpha$-syn PFFs treatment (Fig. 5K), but there was no statistical difference between the ipsilateral and contralateral sides of the globus pallidus (Fig. 6K). In comparison, microglia in other brain regions, such as the caudate nucleus (Supplementary Fig. 4a), putamen (Supplementary Fig. 4b), prefrontal cortex (Supplementary Fig. 4c), and hippocampus (Supplementary Fig. 4d), exhibited much less iron deposition. These data indicate that iron deposition is enriched specifically in the substantia nigra and globus pallidus and at the cellular level, specifically in the microglia.

\section{Expression of iron-related proteins in dopaminergic neurons}

As iron deposition increased in the substantia nigra but the deposits were mostly localized in microglia rather than dopaminergic neurons, we examined how microglia with a high iron content might affect dopaminergic neurons. Using double immunofluorescence staining with antibodies specifically against iron-related proteins and $\mathrm{TH}$, we observed altered expression of iron-related proteins in dopaminergic neurons. Moreover, TF, TFR1, TFR2, and FPn all partially colocalized with $\mathrm{TH}$ (Fig. 7A-D). Compared with the control group, iron- 


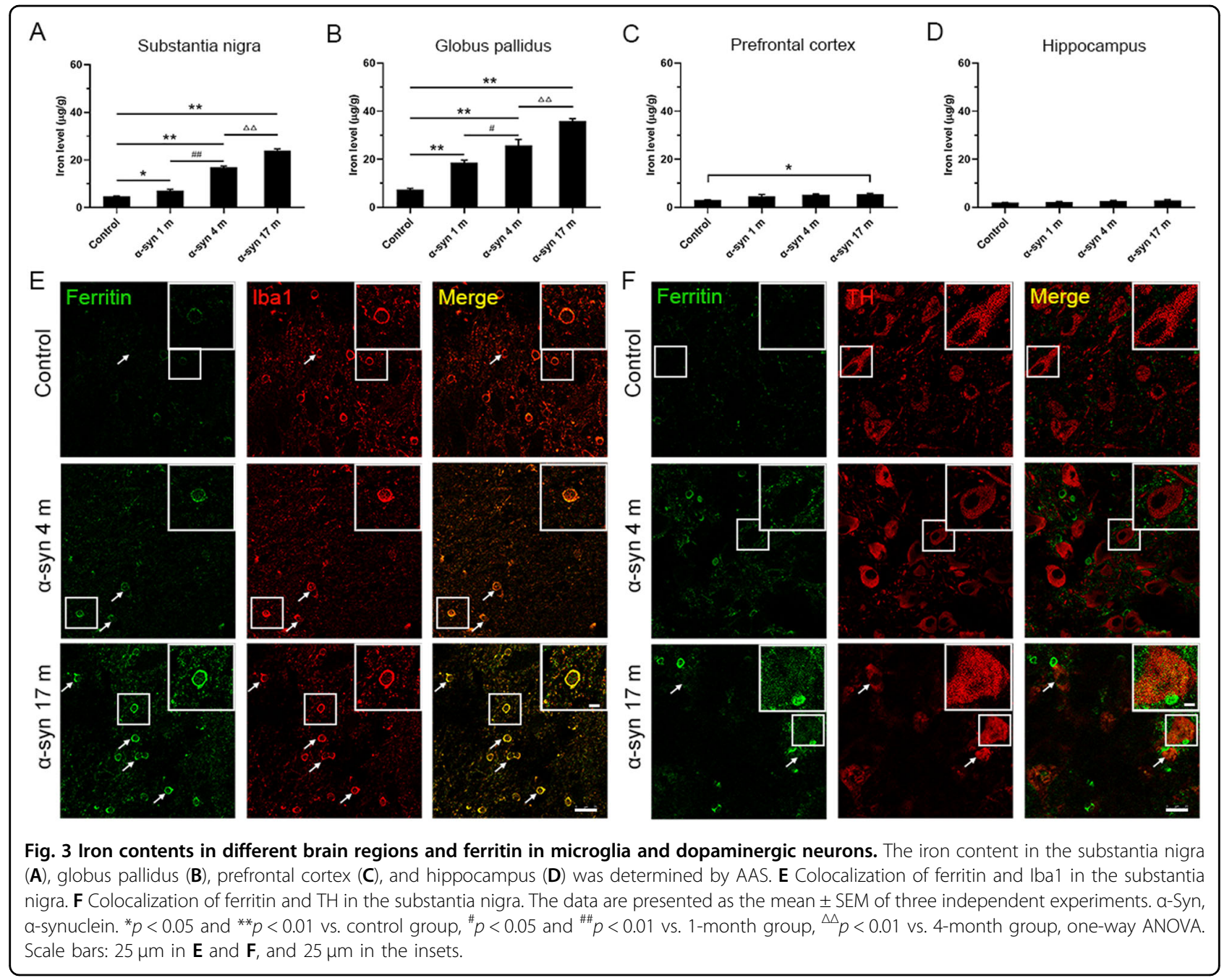

related proteins in dopaminergic neurons in the substantia nigra appeared increased at the 4- and 17-month time points after $\alpha$-syn PFFs treatments and many THpositive cells were also positive for TF (Fig. 7E), TFR1 (Fig. 7F), TFR2 (Fig. 7G), and FPn (Fig. 7H).

No dopaminergic neuronal loss in the substantia nigra, but neurodegenerative terminals in the putamen

Dopaminergic neuronal cell loss and $\alpha$-syn aggregation in the substantia nigra are characteristic features of PD. To examine effects of dopaminergic neurons in response to $\alpha$-syn PFFs administrations via the olfactory epithelium, we quantified dopaminergic neurons in the substantia nigra (Fig. 8A, B) and nerve terminals in the putamen (Fig. 8C-M). Stereological quantitative analyses showed no difference in number of dopaminergic neurons among the different groups (Fig. 8B). Very interestingly, a progressive loss of dopaminergic (TH-positive) terminals was detected in the dorsolateral part of the putamen (Fig. $8 \mathrm{~K}, \mathrm{~L}$ ). The overall density of $\mathrm{TH}$-positive terminals was progressively reduced, and many of the terminals showed signs of damage, expressed as $\mathrm{TH}$-containing axonal swellings (arrows in Fig. 8D-J). Likewise, there was no statistical difference between the ipsilateral and contralateral sides (Fig. 8M).

We, at last, examined whether treatment with $\alpha$-syn had any effects on $\alpha$-syn aggregation and phosphorylation. We barely observed any phosphorylated (pS129) $\alpha$-syn-positive neurons in the substantia nigra of the control (PBS) group (Supplementary Fig. 5a), but we detected sparsely distributed pS129- $\alpha$-syn-positive neurons in the substantia nigra in response to $\alpha$-syn PFFs treatment (Supplementary Fig. 5b-d). $\alpha$-Syn immunoreactivity appeared in diffuse pattern, no typical LB nor Lewy neurites were detected (Supplementary Fig. 5b-d). No distinct phosphorylated $\alpha$-syn-positive neurons nor terminals were observed in the putamen in the 1-, 4-, and 17-month groups after the administration of $\alpha$-syn PFFs (Supplementary Fig. $5 \mathrm{f}-\mathrm{h}$ ), which was similar to the control group (Supplementary Fig. 5e). Moreover, we also performed 


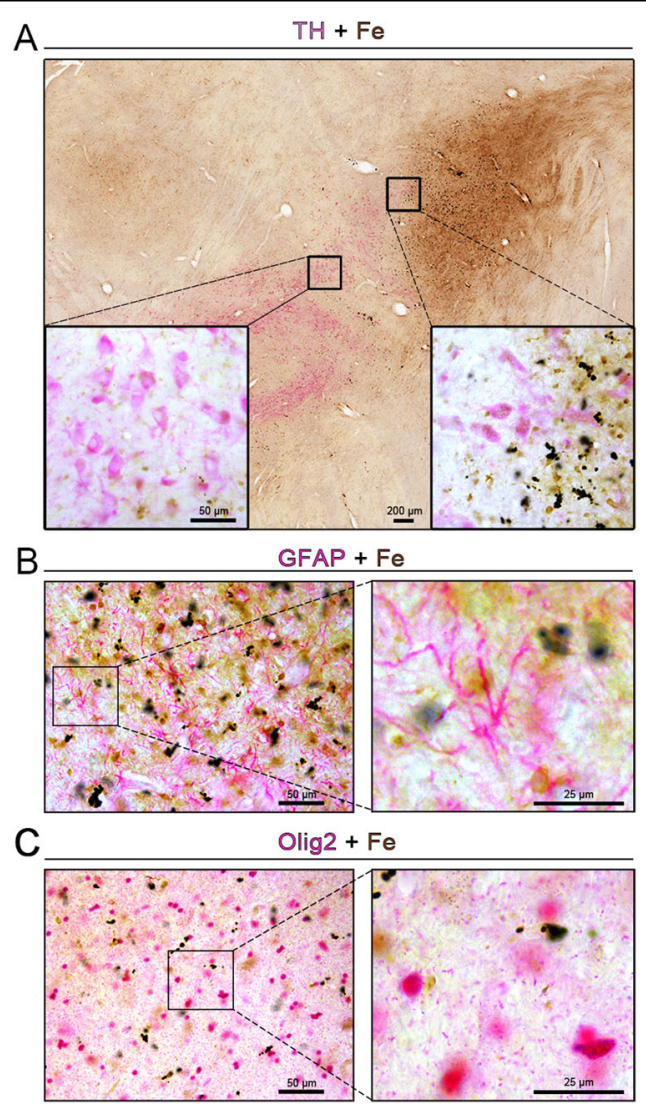

Fig. 4 Cellular localization of iron deposits in the substantia nigra. A-C Double-labeling of chemically stained iron ions (dark brown) along with immunohistochemically stained with TH (A), GFAP (B), or Olig2 (C) antibody (magenta) showed heavy iron deposition 17 months after treatment with a-syn PFFs. The inset images depict the enlarged small boxes in $\mathbf{A}-\mathbf{C}$. Scale bars: $200 \mu \mathrm{m}$ in $\mathbf{A}$ and $50 \mu \mathrm{m}$ in the inset; $50 \mu \mathrm{m}$ in $\mathbf{B}, \mathbf{C}$, and $25 \mu \mathrm{m}$ in the enlarged images.

immunohistochemical staining with $\alpha$-syn or phosphorylated $\alpha$-syn antibodies and also co-stained with iron chemical (Perl's) staining to examine whether $\alpha$-syn colocalize with iron deposition in the substantia nigra. It was evident that iron depositions were largely present in the microglia-like cells (as shown Figs. 5 and 6), whereas $\alpha$-syn appeared as fine granules reflecting a localization in nerve terminal structures. We only observed little overlapping between $\alpha$-syn and iron deposition (Supplementary Fig. 6a). Interestingly, we observed to a certain extent overlapping between phosphorylated $\alpha$-syn and iron deposition in the 4- and 17-month group after $\alpha$-syn PFF treatments (Supplementary Fig. 6b).

\section{No behavioral alterations after the $a$-syn PFFs treatment}

To examine whether the $\alpha$-syn PFFs treatment may induce behavioral changes, we performed two types of behavioral tests. One was the delay match-to-sample (DMTS), which evaluated the short term memory of cognitive behavior. The other was the pick-up test (PUT), which quantitatively measured the fine hand motor performance skills. Due to the long-term (for 6 months) training process, we only performed the two tests in the monkeys 17 months after $\alpha$-syn PFFs delivery. Interestingly, we did not observe significant alterations on either DMTS (Supplementary Fig. 7a, b) or PUT tests (Supplementary Fig. 7c, d).

\section{Discussion}

Iron accumulates to a much higher extent in specific brain regions in various neurodegenerative diseases ${ }^{33-36}$. The functional impairment of dopaminergic neurons in the nigra-striatal system caused by high iron in the brain has drawn considerable attention in the field of PD research ${ }^{37,38}$. Dopaminergic neuron degeneration is evident in the brains of PD patients and iron accumulation is usually associated with this cytopathic effect ${ }^{39,40}$. Here we present data confirming the presence of excessive iron deposition in specific brain regions in monkeys, mimicking the human situation.

During the course of PD, dopaminergic neurons gradually lose their function, eventually leading to cell death. Iron plays a key role in this process; thus, the excessive deposition of iron may be involved in the degenerative death process of dopaminergic neurons in $\mathrm{PD}^{32,41,42}$. In the present study, up to the 1,4 , and 17 months after the treatment with the $\alpha$-syn PFFs, we did not observe dopaminergic neuron loss in the substantia nigra, nor motor and cognitive dysfunction (Supplementary Fig. 7) in consistent with the previous report ${ }^{43}$, but rather, only detected signs of dopaminergic neurodegeneration, i.e., reduced TH intensity and axonal swelling in the putamen $^{44-46}$. Interestingly, an increase in the iron content was detected in the brains of monkeys in the PD model groups and further studies revealed that the increased iron was mainly deposited in the substantia nigra and globus pallidus, which are also the brain regions that exhibit lesions in PD patients ${ }^{47-51}$. These results provide evidence supporting the tropism of $\alpha$-syn PFFs in the substantia nigra $^{52-54}$, which leads us to speculate that iron deposition precedes dopaminergic neuron loss. However, further investigation on the underlying mechanisms on how $\alpha$-syn aggregates causes PD and the relationship between $\alpha$-syn aggregation and iron metabolism is warranted. In fact, some human PD brain regions, such as the substantia nigra and striatum, exhibit excessive iron accumulation ${ }^{55,56}$. Similarly, iron deposits are present in the substantia nigra in 6-OHDA-induced PD rat models $^{57,58}$. In addition, methyl-4-phenyl-1, 2, 3, 6-tetrahydropyridine, a neurotoxin, can induce PD-like symptoms and iron is involved in its conversion to the toxic metabolite 1-methyl-4-phenylpyridinium ion ${ }^{59}$; during conversion, the induced cellular damage and neurotoxicity are considered to be associated with 


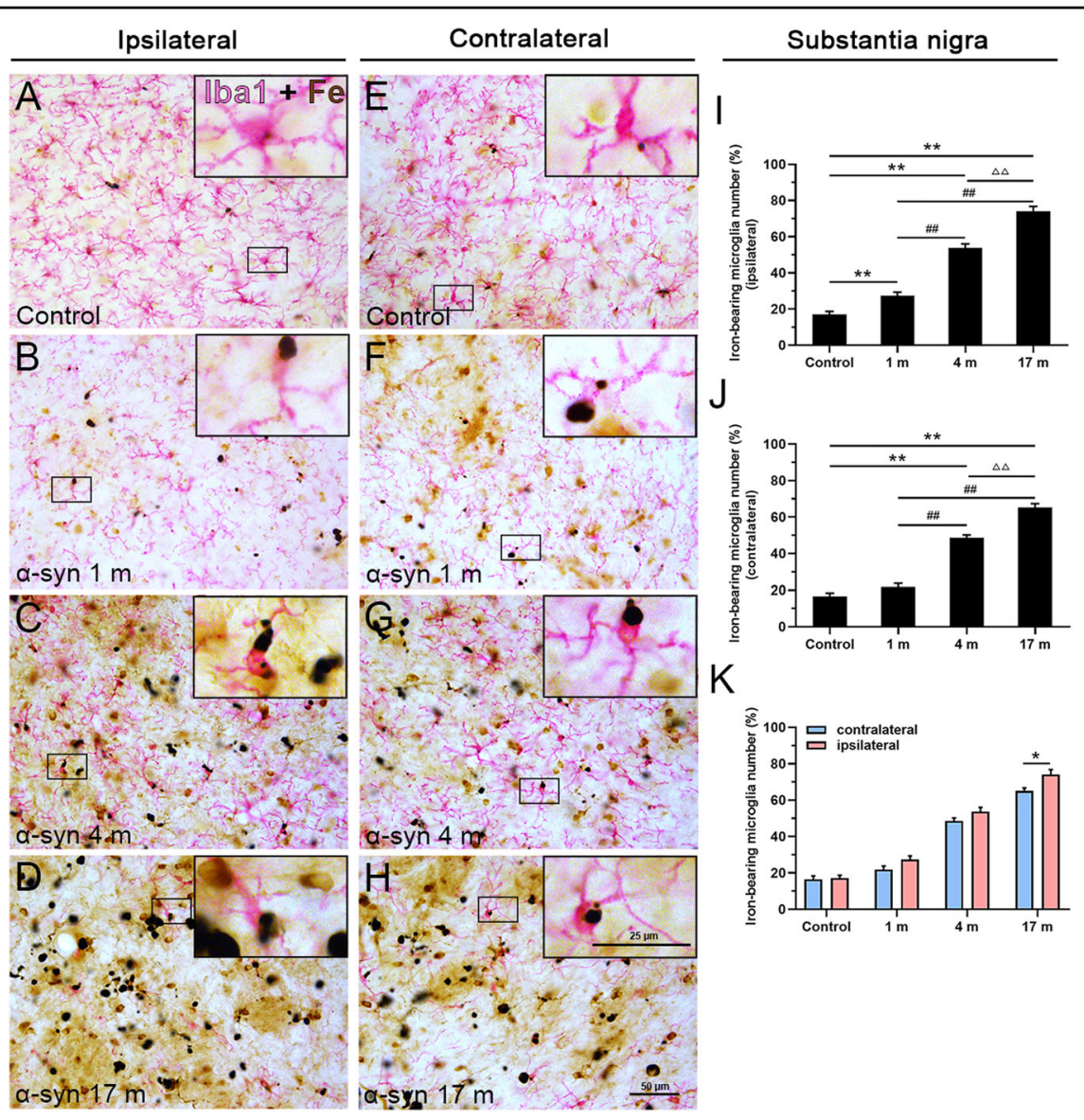

Fig. 5 Cellular localization of iron deposits in microglia of the substantia nigra. Double-labeling of chemically stained iron ions (dark brown) along with immunohistochemical staining with an Iba1 antibody (magenta) showed overlapping of iron deposition on Iba1-positive microglia in the control group (A) and 1, 4, and 17 months after the treatment with a-syn PFFs in the ipsilateral side (B-D), control group (E), and 1, 4, and 17 months after treatment with a-syn PFFs in the contralateral side $(\mathbf{F}-\mathbf{H})$. I-K Quantitative analyses of iron deposition in microglia in the substantia nigra (ipsilateral side: $\mathbf{I}$; contralateral side: $\mathbf{J}$; both sides: $\mathbf{K}$ ). a-Syn, a-synuclein. The data are presented as the mean \pm SEM of three independent experiments. ${ }^{* *} p<0.01$ vs. control group, ${ }^{\# \#} p<0.01$ vs. 1 -month group, ${ }^{\Delta \Delta} p<0.01$ vs. 4 -month group, one-way ANOVA (I, J). ${ }^{*} p<0.05$ vs. contralateral group, Tukey's multiple comparisons test after two-way ANOVA (K). Scale bars: $50 \mu \mathrm{m}$ in $\mathbf{A}-\mathbf{H}$ and $25 \mu \mathrm{m}$ in the insets.

changes in iron and iron metabolism ${ }^{60}$. Importantly, $\alpha-$ syn contains divalent metal ions, including iron-binding sites $^{61}$. Thus, it is conceivable that exposure to $\alpha$-syn PFFs may cause iron deposition, at least partially, by altering iron metabolism in the brain areas affected in PD, eventually resulting in dopaminergic neuron damage.

The reasoning proposed here is supported by Oakley et al. ${ }^{16}$, who examined fresh specimens of the substantia nigra from PD patients using sensitive and specific wavelength dispersive electron probe X-ray microanalysis coupled with cathodoluminescence spectroscopy, and found that the iron content in the neurons in the substantia nigra was increased, suggesting that increased neuronal iron in PD may be a primary event leading to $\alpha$-syn aggregation. In the present study, we observed that iron mostly accumulates in microglia rather than in dopaminergic neurons. Furthermore, dopaminergic neuron death was not detected in the monkeys treated with $\alpha$-syn PFFs. Although impaired microglia have been reported to be closely associated with iron deposition ${ }^{62,63}$, in the present study, most microglia in this region were activated. The excessive activation of microglia could cause neurotoxicity and produce numerous neuroimmune proinflammatory factors that lead to oxidative stress, further promoting dopaminergic neuron damage ${ }^{41,64}$.

The increased iron concentration in PD brains may be due to the abnormal regulation of iron metabolism in neurons and glia ${ }^{65}$. TF is an important iron-binding, transport and storage protein and an important regulatory protein of iron metabolism in the body. Both TF and TFR are involved in maintaining the balance of iron metabolism, and iron transport by TF/TFR is the main source of iron in tissues. An increase in TF/TFR leads to an increase in the intracellular iron content $^{13}$. It has been 


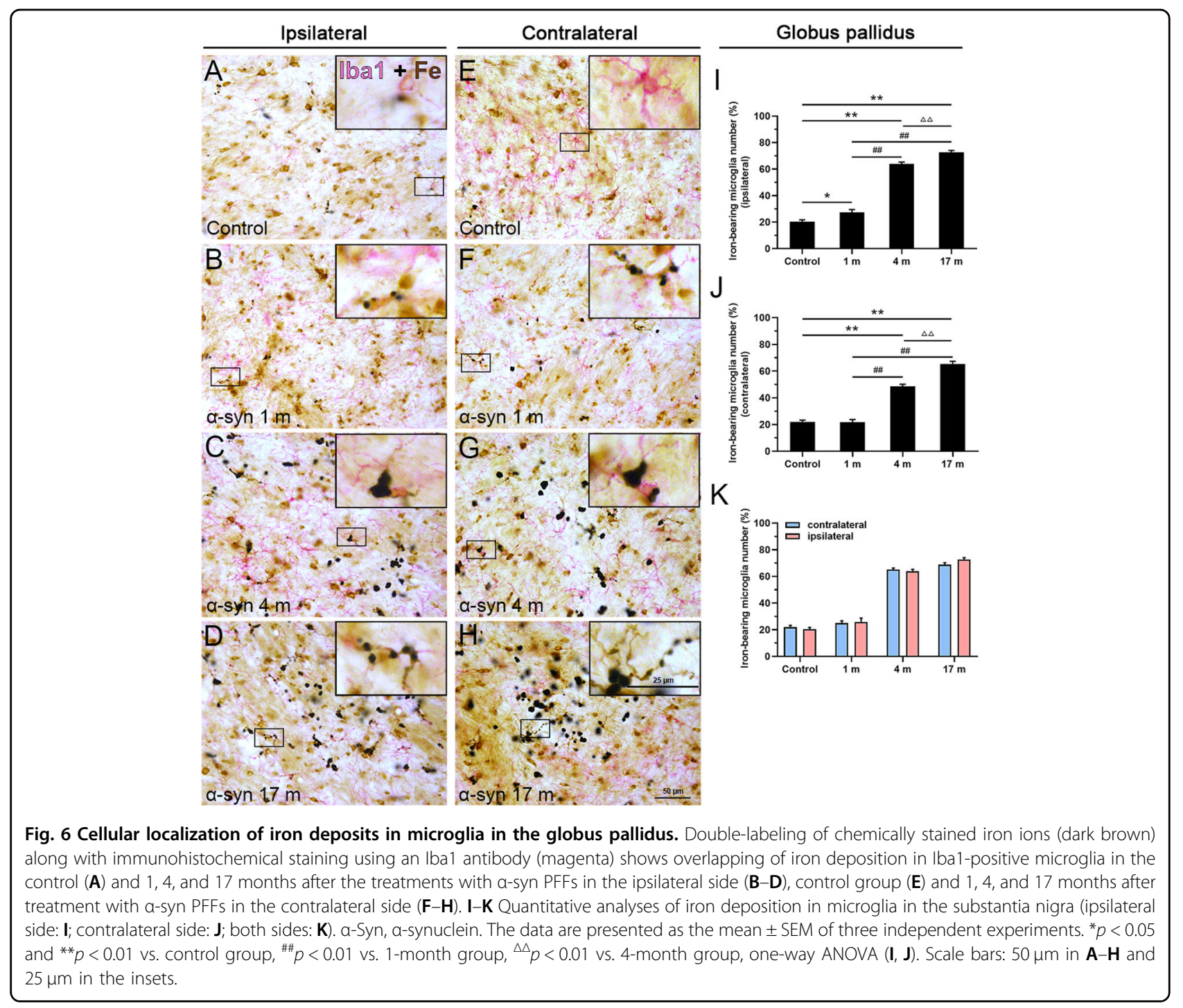

demonstrated by experimental approaches that the TF/ TFR2 complex may have an important role in the etiology of $\mathrm{PD}^{66,67}$. Currently, FPn is the only known transmembrane iron transporter and FPn dysfunction causes disturbances in iron metabolism ${ }^{68}$. In the present study, an increase in the TF/TFR1/TFR2/FPn proteins was detected in the substantia nigra, leading to an increase in the intracellular iron ion content and iron accumulation ${ }^{13,68}$. Moreover, we observed cell-type-dependent iron enrichment in microglia and low iron abundance in dopaminergic neurons in the substantia nigra. Microglia maintain iron homeostasis in the brain mainly by sequestering and storing iron within ferritin ${ }^{69}$, and microglia activation is an important cause of high iron concentrations in the brain $^{70}$. As expected, we also detected an increase in the immunoreactivity of ferritin in $\alpha$-syn PFFs-treated microglia, which is related to the pathogenesis of degeneration of nigrostriatal dopamine neurons in $\mathrm{PD}^{18}$.
Therefore, it is conceivable that the iron metabolismrelated proteins TFR/TF and FPn modulate iron transport between different types of cells in PD-affected brain regions in response to $\alpha$-syn PFFs treatment, which could impair the normal transport of iron by iron-related proteins in the nigra-striatal system, and the iron deposition in microglia may indirectly cause dysfunction in dopaminergic neurons in $\mathrm{PD}^{71,72}$.

What is the consequence of increased iron deposition in microglia rather than dopaminergic neurons in the monkeys treated with $\alpha$-syn PFFs treatment? Although we did not observe clear dopaminergic neuronal loss, we detected an increased cellular level of iron-related proteins, such as TFR1/TFR2/TF and FPn, in dopaminergic neurons. Therefore, we propose two possible underlying mechanisms. First, exposure to $\alpha$-syn PFFs treatment may initiate the process of neuroinflammation by triggering iron accumulation in microglia and then inducing cascade responses 


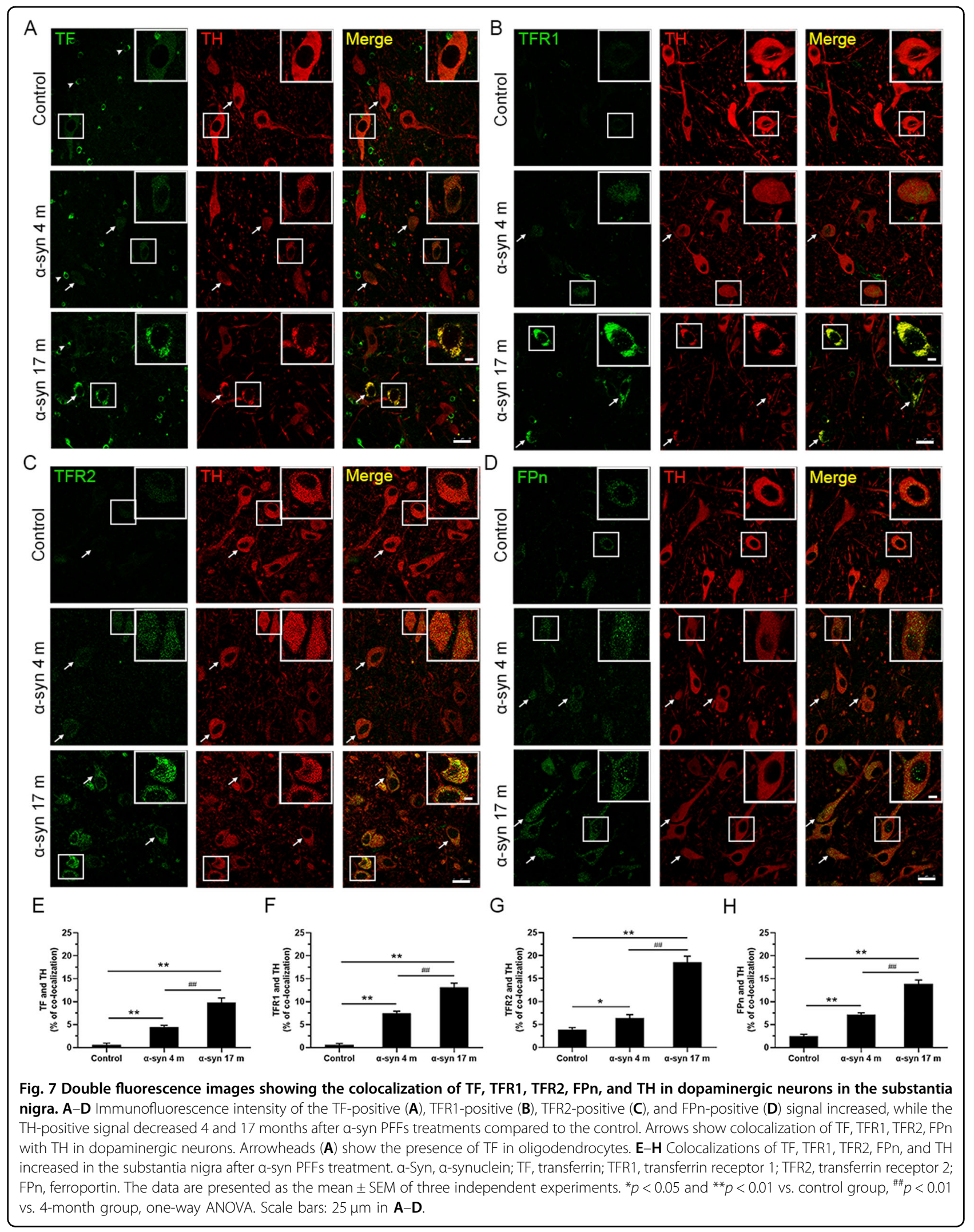




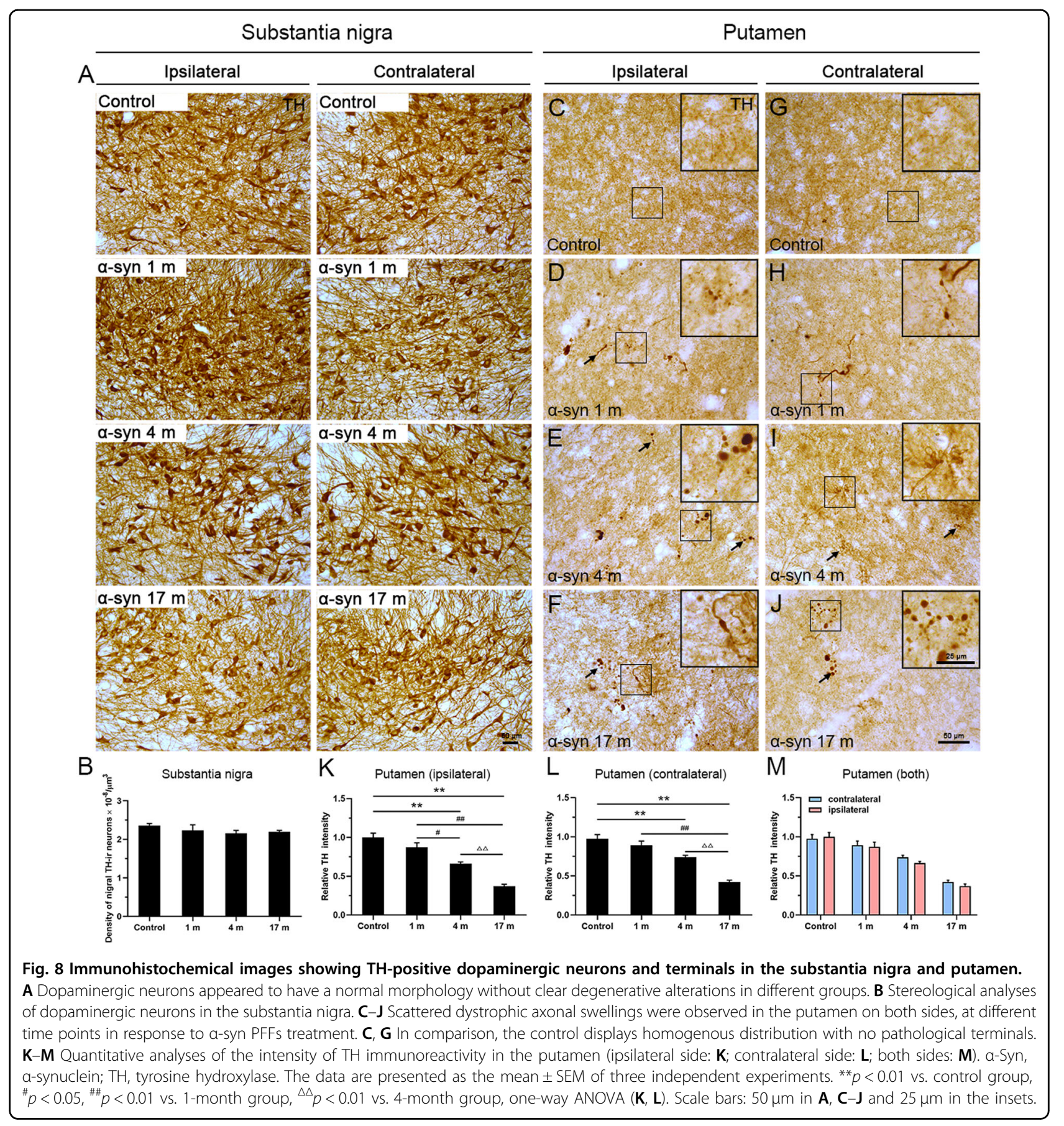

between iron deposition and microglial activation, generating hydrogen peroxide and hydroxyl radicals, releasing proinflammatory factors and leading to $\alpha$-syn aggregation, dopaminergic neuron toxicity, and degeneration ${ }^{22,73}$. Second, under both physiological and pathophysiological conditions, iron continually moves across neurons, microglia, and astrocytes ${ }^{74}$. Although it has been shown that the level of iron is elevated in dopaminergic neurons in $\mathrm{PD}^{16}$, we did not observe a similar phenomenon in dopaminergic neurons, but we detected robust iron deposits in microglia. Two reasons may explain this inconsistency. Most, if not all, studies using PD patient materials were obtained from patients with an advanced stage of PD, while the current monkey model treated with $\alpha$-syn PFFs treatment only mimicked the early stage and even the prodromal stage of PD. It is conceivable that iron deposition first appears in microglia in the substantia nigra, and then, with the progression of PD pathology, the iron in microglia may 
translocate from nearby microglia to dopaminergic neurons and be regulated by iron-related proteins present in dopaminergic neurons. Moreover, no dopaminergic neuronal loss in the substantia nigra, decreased $\mathrm{TH}$ immunoreactivity in the putamen, together with mildly and sparsely distributed phosphorylated $\alpha$-syn in dopaminergic neurons in the substantia nigra, but absence in the putamen reinforced the claim that iron deposition in microglia precedes dopaminergic neuronal degeneration. In summary, our findings indicated that iron deposition in the nigra-striatal system of monkeys precedes dopaminergic neuronal degeneration after nasal administration of exogenous $\alpha$-syn PFFs. Based on the present study, the iron deposition in microglia and abnormal iron transport-related proteins appear to involve in the pathogenesis of PD and may become a tool for the early diagnosis of $\mathrm{PD}$, such as magnetic resonance imaging of iron contents. The modulation of abnormal iron metabolism may be a potential therapeutic strategy for PD.

\section{Acknowledgements}

We thank members of the Li laboratory for their intellectual inputs.

\begin{abstract}
Author details
'Institute of Neuroscience, College of Life and Health Sciences, Northeastern University, Shenyang 110819, China. 'Beijing Key Laboratory of Parkinson's Disease, National Clinical Research Center for Geriatric Disorders, Department of Neurobiology, Xuanwu Hospital of Capital Medical University, Beijing 100053, China. 'Laboratory of Neurodegenerative Diseases, CNRS and Institut François Jacob (MIRCen), CEA, Fontenay-aux-Roses 92260, France. ${ }^{4}$ Department of Histology, Chongqing Medical University, Chongqing 400000, China. ${ }^{5}$ Unit of Neurodegenerative Diseases and Repair, Institute of Health Sciences, China Medical University, Shenyang 110112, China. ${ }^{6}$ Neural Plasticity and Repair Unit, Wallenberg Neuroscience Center, Lund University, Lund 22184, Sweden
\end{abstract}

\section{Author contributions}

J.J.G., C.G., and J.Y.L. designed the study. J.J.G., C.G., P.C., and J.Y.L. performed the acquisition of data, and analyzed and interpreted the results. F.Y. and P.C. collected tissues. Y.T., X.L., and J.T. performed the stereological analyses. J.J.G., C.G., and J.Y.L. wrote the manuscript. L.B., R.M., D.Y.S., Y.L., and W.L. had critically revised it. All authors read and approved the final version of the manuscript.

\section{Funding}

This work was supported by the National Key Research and Development Program of China [2018YFA0108500], the National Natural Science Foundation [81430025, 31970967, 81701265, 31800898, U1801681, and 31472056], the Key Field Research Development Program of Guangdong Province

[2018B030337001], the Guangxi Sci-Tech project [Guike AD17195075], the Swedish Research Council (K2015-61X-22297-03-4), EU-JPND (a-SynProtec) and EU-JPND (REfrAME), EU H2020-MSCA-ITN-2016 Syndegen, the Strong Research Environment MultiPark (Multidisciplinary research on Parkinson's disease), Parkinsonfonden, Torsten Söderbergs Foundation, and Olle Engkvist Byggmästare Foundation. L.B. and R.M. were supported by the CNRS and the EC Joint Programme on Neurodegenerative Diseases TransPathND (ANR-17JPCD-0002-02) and Protest-70 (ANR-17-JPCD-0005-01). Open Access funding provided by Lund University.

\section{Ethics statement}

All procedures were reviewed and approved by the Institutional Animal Care and Use Committee (IACUC) of Wincon TheraCells Biotechnologies Co., Ltd (Wincon) in Nanning, Guangxi, China, which is fully accredited by the Association for Assessment and Accreditation of Laboratory Animal Care (AAALAC).

\section{Conflict of interest}

The authors declare that they have no conflict of interest.

\section{Publisher's note}

Springer Nature remains neutral with regard to jurisdictional claims in published maps and institutional affiliations.

Supplementary Information accompanies this paper at (https://doi.org/ 10.1038/s41419-020-03369-x).

Received: 19 August 2020 Revised: 16 December 2020 Accepted: 17 December 2020

Published online: 13 January 2021

\section{References}

1. Jiang, P. \& Dickson, D. W. Parkinson's disease: experimental models and reality. Acta Neuropathol. 135, 13-32 (2018).

2. Barboza, J. L., Okun, M. S. \& Moshiree, B. The treatment of gastroparesis, constipation and small intestinal bacterial overgrowth syndrome in patients with Parkinson's disease. Expert. Opin. Pharmacother. 16, 2449-2464 (2015).

3. Marinus, J., Zhu, K., Marras, C., Aarsland, D. \& van Hilten, J. J. Risk factors for nonmotor symptoms in Parkinson's disease. Lancet Neurol. 17, 559-568 (2018).

4. Aarsland, D. et al. Cognitive decline in Parkinson disease. Nat. Rev. Neurol. 13, 217-231 (2017).

5. Jankovic, J. Parkinson's disease: clinical features and diagnosis. J. Neurol. Neurosurg. Psychiatry 79, 368-376 (2008).

6. Braak, H. \& Tredici, D. K. Neuropathological staging of brain pathology in sporadic Parkinson's disease: separating the wheat from the Chaff. J. Parkinson's Dis. 7, S71-s85 (2017).

7. Rayman, J. B. \& Kandel, E. R. Erratum: Functional prions in the brain. Cold Spring Harb. Perspect. Biol 9, a033597 (2017).

8. Vaccari, C., El Dib, R., Gomaa, H., Lopes, L. C. \& de Camargo, J. L. Paraquat and Parkinson's disease: a systematic review and meta-analysis of observational studies. J. Toxicol. Environ. Health Part B Crit. Rev. 22, 172-202 (2019).

9. Langley, J. et al. Reproducible detection of nigral iron deposition in 2 Parkinson's disease cohorts. Mov. Disord. 34, 416-419 (2019).

10. Graham, J. M., Paley, M. N., Grunewald, R. A., Hoggard, N. \& Griffiths, P. D. Brain iron deposition in Parkinson's disease imaged using the PRIME magnetic resonance sequence. Brain J. Neurol. 123, 2423-2431 (2000). Pt 12.

11. Farrall, A. J. \& Wardlaw, J. M. Blood-brain barrier: ageing and microvascular disease-systematic review and meta-analysis. Neurobiol. Aging 30, 337-352 (2009).

12. Conde, J. R. \& Streit, W. J. Microglia in the aging brain. J. Neuropathol. Exp. Neurol. 65, 199-203 (2006).

13. Connor, J. R., Menzies, S. L., Burdo, J. R. \& Boyer, P. J. Iron and iron management proteins in neurobiology. Pediatr. Neurol. 25, 118-129 (2001).

14. Ke, Y. \& Qian, Z. M. Brain iron metabolism: neurobiology and neurochemistry. Prog. Neurobiol. 83, 149-173 (2007)

15. Zecca, L., Youdim, M. B., Riederer, P., Connor, J. R. \& Crichton, R. R. Iron, brain ageing and neurodegenerative disorders. Nat. Rev. Neurosci. 5, 863-873 (2004).

16. Oakley, A. E. et al. Individual dopaminergic neurons show raised iron levels in Parkinson disease. Neurology 68, 1820-1825 (2007).

17. Andersen, H. H., Johnsen, K. B. \& Moos, T. Iron deposits in the chronically inflamed central nervous system and contributes to neurodegeneration. Cell. Mol. Life Sci. 71, 1607-1622 (2014).

18. Jellinger, K., Paulus, W., Grundke, I. I., Riederer, P. \& Youdim, M. B. Brain iron and ferritin in Parkinson's and Alzheimer's diseases. J. Neural Transm. Parkinson's Dis. Dement. Sect. 2, 327-340 (1990).

19. McGeer, P. L., Itagaki, S., Boyes, B. E. \& McGeer, E. G. Reactive microglia are positive for HLA-DR in the substantia nigra of Parkinson's and Alzheimer's disease brains. Neurology 38, 1285-1291 (1988).

20. Langston, J. W. et al. Evidence of active nerve cell degeneration in the substantia nigra of humans years after 1-methyl-4-phenyl-1,2,3,6-tetrahydropyridine exposure. Ann. Neurol. 46, 598-605 (1999).

21. Uversky, V. N., Li, J. \& Fink, A. L. Metal-triggered structural transformations, aggregation, and fibrillation of human alpha-synuclein. A possible molecular NK between Parkinson's disease and heavy metal exposure. J. Biol. Chem. 276, 44284-44296 (2001).

22. el-Agnaf, O. M. \& Irvine, G. B. Aggregation and neurotoxicity of alpha-synuclein and related peptides. Biochem. Soc. Trans. 30, 559-565 (2002).

23. Castellani, R. J., Siedlak, S. L., Perry, G. \& Smith, M. A. Sequestration of iron by Lewy bodies in Parkinson's disease. Acta Neuropathol. 100, 111-114 (2000). 
24. Faucheux, B. A. et al. Neuromelanin associated redox-active iron is increased in the substantia nigra of patients with Parkinson's disease. J. Neurochem. 86, 1142-1148 (2003)

25. Wang, J. Y. et al. Meta-analysis of brain iron levels of Parkinson's disease patients determined by postmortem and MRI measurements. Sci. Rep. 6, 36669 (2016).

26. Ward, R. J., Zucca, F. A., Duyn, J. H., Crichton, R. R. \& Zecca, L. The role of iron in brain ageing and neurodegenerative disorders. Lancet Neurol. 13, 1045-1060 (2014).

27. Holmqvist, S. et al. Direct evidence of Parkinson pathology spread from the gastrointestinal tract to the brain in rats. Acta Neuropathol. 128, 805-820 (2014).

28. Langley, J., Huddleston, D. E., Sedlacik, J., Boelmans, K. \& Hu, X. P. Parkinson's disease-related increase of $T 2^{*}$-weighted hypointensity in substantia nigra pars compacta. Mov. Disord. 32, 441-449 (2017).

29. Sian-Hulsmann, J., Mandel, S., Youdim, M. B. \& Riederer, P. The relevance of iron in the pathogenesis of Parkinson's disease. J. Neurochem. 118, 939-957 (2011).

30. Jenner, P. \& Olanow, C. W. Understanding cell death in Parkinson's disease. Ann. Neurol. 44, S72-S84 (1998).

31. Fahn, S. \& Cohen, G. The oxidant stress hypothesis in Parkinson's disease: evidence supporting it. Ann. Neurol. 32, 804-812 (1992).

32. Kaur, D. et al. Genetic or pharmacological iron chelation prevents MPTPinduced neurotoxicity in vivo: a novel therapy for Parkinson's disease. Neuron 37, 899-909 (2003).

33. Dexter, D. T. et al. Increased nigral iron content in postmortem parkinsonian brain. Lancet 2, 1219-1220 (1987).

34. Hirsch, E. C., Brandel, J. P., Galle, P., Javoy-Agid, F. \& Agid, Y. Iron and aluminum increase in the substantia nigra of patients with Parkinson's disease: an X-ray microanalysis. J. Neurochem. 56, 446-451 (1991)

35. Riederer, P. et al. Transition metals, ferritin, glutathione, and ascorbic acid in parkinsonian brains. J. Neurochem. 52, 515-520 (1989).

36. Lei, P., Ayton, S. \& Bush, A. I. The essential elements of Alzheimer's disease. J. Biol. Chem. https://doi.org/10.1074/jbc.REV120.008207 (2020).

37. Good, P. F., Olanow, C. W. \& Perl, D. P. Neuromelanin-containing neurons of the substantia nigra accumulate iron and aluminum in Parkinson's disease: a LAMMA study. Brain Res. 593, 343-346 (1992).

38. Rouault, T. A. Systemic iron metabolism: a review and implications for brain iron metabolism. Pediatr. Neurol. 25, 130-137 (2001).

39. Daglas, M. \& Adlard, P. A. The involvement of iron in traumatic brain injury and neurodegenerative disease. Front. Neurosci. 12, 981 (2018).

40. Grassi, D. et al. Identification of a highly neurotoxic alpha-synuclein species inducing mitochondrial damage and mitophagy in Parkinson's disease. Proc. Natl Acad. Sci. USA 115, E2634-e2643 (2018).

41. Peng, J., Peng, L., Stevenson, F. F., Doctrow, S. R. \& Andersen, J. K. Iron and paraquat as synergistic environmental risk factors in sporadic Parkinson's disease accelerate age-related neurodegeneration. J. Neurosci. 27, 6914-6922 (2007).

42. Faucheux, B. A. et al. Expression of lactoferrin receptors is increased in the mesencephalon of patients with Parkinson disease. Proc. Natl Acad. Sci. USA 92, 9603-9607 (1995).

43. Beck, G. et al. Role of striatal $\triangle F o s B$ in I-Dopa-induced dyskinesias of parkinsonian nonhuman primates. Proc. Natl Acad. Sci. USA 116, 18664-18672 (2019).

44. Faustini, G. et al. Synapsin III deficiency hampers alpha-synuclein aggregation, striatal synaptic damage and nigral cell loss in an AAV-based mouse model of Parkinson's disease. Acta Neuropathol. 136, 621-639 (2018).

45. Galvin, J. E. Interaction of alpha-synuclein and dopamine metabolites in the pathogenesis of Parkinson's disease: a case for the selective vulnerability of the substantia nigra. Acta Neuropathol. 112, 115-126 (2006).

46. Michel, P. P., Hirsch, E. C. \& Hunot, S. Understanding dopaminergic cell death pathways in Parkinson disease. Neuron 90,675-691 (2016).

47. Jellinger, K. A. A critical reappraisal of current staging of Lewy-related pathology in human brain. Acta Neuropathol. 116, 1-16 (2008).

48. Jellinger, K. A. Alpha-synuclein pathology in Parkinson's and Alzheimer's disease brain: incidence and topographic distribution-a pilot study. Acta Neuropathol. 106, 191-201 (2003).

49. Warren, O. C. et al. Gene delivery of neurturin to putamen and substantia nigra in Parkinson disease: a double-blind, randomized, controlled trial. Ann. Neurol. 78, 248-257 (2015).
50. Bohnen, N. I. et al. Frequency of cholinergic and caudate nucleus dopaminergic deficits across the predemented cognitive spectrum of Parkinson disease and evidence of interaction effects. JAMA Neurol. 72, 194-200 (2015).

51. Ramirez, Z. A. \& Ostrem, J. L. Globus pallidus interna or subthalamic nucleus deep brain stimulation for Parkinson disease: a review. JAMA Neurol. 75 , 367-372 (2018)

52. Chu, Y. et al. Intrastriatal alpha-synuclein fibrils in monkeys: spreading, imaging and neuropathological changes. Brain 142, 3565-3579 (2019).

53. Recasens, A. et al. Lewy body extracts from Parkinson disease brains trigger asynuclein pathology and neurodegeneration in mice and monkeys. Ann. Neurol. 75, 351-362 (2014).

54. Shimozawa, A. et al. Propagation of pathological a-synuclein in marmoset brain. Acta Neuropathol. Commun. 5, 12 (2017).

55. Chen, Q. et al. Iron deposition in Parkinson's disease by quantitative susceptibility mapping. BMC Neurosci. 20, 23 (2019).

56. Lewis, M. M. et al. Higher iron in the red nucleus marks Parkinson's dyskinesia. Neurobiol. Aging 34, 1497-1503 (2013).

57. Zhu, Y. et al. Iron accumulation and microglia activation contribute to substantia nigra hyperechogenicity in the 6-OHDA-induced rat model of Parkinson's disease. Parkinsonism Relat. Disord. 36, 76-82 (2017).

58. Olmedo, D. S. et al. An altered blood-brain barrier contributes to brain iron accumulation and neuroinflammation in the 6-OHDA rat model of Parkinson's disease. Neuroscience 362, 141-151 (2017).

59. Di Monte, D. A., Schipper, H. M., Hetts, S. \& Langston, J. W. Iron-mediated bioactivation of 1-methyl-4-phenyl-1,2,3,6-tetrahydropyridine (MPTP) in glial cultures. Glia 15, 203-206 (1995).

60. Hare, D. J., Adlard, P. A., Doble, P. A. \& Finkelstein, D. I. Metallobiology of 1methyl-4-phenyl-1,2,3,6-tetrahydropyridine neurotoxicity. Metallomics 5, 91-109 (2013).

61. Binolfi, A. et al. Interaction of alpha-synuclein with divalent metal ions reveals key differences: a link between structure, binding specificity and fibrillation enhancement. J. Am. Chem. Soc. 128, 9893-9901 (2006).

62. Hametner, S. et al. Iron and neurodegeneration in the multiple sclerosis brain. Ann. Neurol. 74, 848-861 (2013).

63. Haider, L. et al. Multiple sclerosis deep grey matter: the relation between demyelination, neurodegeneration, inflammation and iron. J. Neurol. Neurosurg. Psychiatry 85, 1386-1395 (2014).

64. Ouchi, Y., Yagi, S., Yokokura, M. \& Sakamoto, M. Neuroinflammation in the living brain of Parkinson's disease. Parkinsonism Relat. Disord. 15, S200-S204 (2009). Suppl 3

65. Qian, Z. M. \& Ke, Y. Brain iron transport. Biol. Rev. Camb. Philos. Soc. 94 1672-1684 (2019).

66. Rhodes, S. L. et al. Pooled analysis of iron-related genes in Parkinson's disease: association with transferrin. Neurobiol. Dis. 62, 172-178 (2014).

67. Mastroberardino, P. G. et al. A novel transferrin/TfR2-mediated mitochondrial iron transport system is disrupted in Parkinson's disease. Neurobiol. Dis. 34, 417-431 (2009).

68. Donovan, A. et al. The iron exporter ferroportin/Slc40a1 is essential for iron homeostasis. Cell Metab. 1, 191-200 (2005).

69. Zhang, X., Surguladze, N., Slagle, W. B., Cozzi, A. \& Connor, J. R. Cellular iron status influences the functional relationship between microglia and oligodendrocytes. Glia 54, 795-804 (2006).

70. Gerlach, M., Double, K. L., Youdim, M. B. \& Riederer, P. Potential sources of increased iron in the substantia nigra of parkinsonian patients. J. Neural Transm. Suppl. 133-142 (2006).

71. Kawai, J. et al. Pallidonigroluysian degeneration with iron deposition: a study of three autopsy cases. Acta Neuropathol. 86, 609-616 (1993).

72. Belaidi, A. A. \& Bush, A. I. Iron neurochemistry in Alzheimer's disease and Parkinson's disease: targets for therapeutics. J. Neurochem. 139 Suppl 1 179-197 (2016).

73. Turnbull, S. et al. alpha-Synuclein implicated in Parkinson's disease catalyses the formation of hydrogen peroxide in vitro. Free Radic. Biol. Med. 30, 1163-1170 (2001).

74. Zucca, F. A., Cupaioli, F. A. \& Zecca, L. The role of iron in neurodegeneration. Neurodegener. Metallostasis Proteostasis 9, 174-211 (2011). 